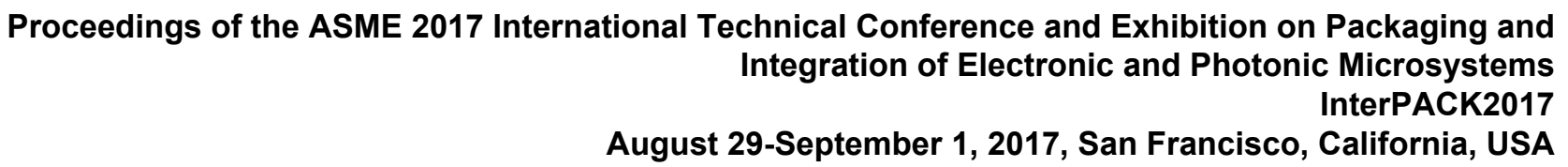

IPACK2017-74178

\title{
SILICA NANOPARTICLE FORMATION BY USING DROPLET-BASED MICROREACTOR
}

\author{
Arsalan Nikdoost \\ Department of Mechanical Engineering \\ Bilkent University \\ Ankara, Turkey, 06800 \\ Email: arsalan.nikdoost@bilkent.edu.tr
}

\author{
Alican Ozkan \\ Department of Mechanical Engineering \\ Bilkent University \\ Ankara, Turkey, 06800 \\ Email: alican.ozkan@bilkent.edu.tr
}

\author{
Yusuf Kelestemur \\ UNAM- Institute of Material \\ Science and Nanotechnology \\ Bilkent University \\ Ankara, Turkey, 06800 \\ Email: \\ kelestemur@bilkent.edu.tr
}

\author{
Hilmi Volkan Demir \\ Department of Electrical and \\ Electronics Engineering \\ UNAM- Institute of Material \\ Science and Nanotechnology \\ Bilkent University \\ Ankara, Turkey, 06800 \\ Email: volkan@bilkent.edu.tr
}

\author{
E. Yegan Erdem \\ Department of Mechanical \\ Engineering \\ UNAM- Institute of Material \\ Science and Nanotechnology \\ Bilkent University \\ Ankara, Turkey, 06800 \\ Email: \\ yeganerdem@bilkent.edu.tr
}

\begin{abstract}
This paper describes a method for the synthesis of silica nanoparticles that can be later used for coating of quantum dots inside a microfluidic reactor. Here, a droplet-based system is used where two reagents were mixed inside the droplets to obtain silica. Particles in the size range of $25 \pm 2.7 \mathrm{~nm}$ were obtained with comparable size distribution to controlled batchwise synthesis methods. This method is suitable to be used later to coat $\mathrm{CdSe}$ nanoparticles inside the microreactor.
\end{abstract}

\section{INTRODUCTION}

Silica is an important material that is used for coating of quantum dots (QD) to prolong the optical and chemical stability as well as to enable the dispersion of the particles in water, which is necessary to use them in biomedical applications [1-4]. The conventional batch-wise synthesis methods such as waterin-oil microemulsions [5] and reverse micelle [6] usually require long processing times (several hours) to synthesize silica. On the other hand, microfluidic reactors are promising candidates to decrease this time to only a few hours. Until now, microfluidic reactors have shown to be great platforms for the synthesis of monodisperse nanoparticles as they can provide precise control over reaction parameters such as temperature, concentration of reagents and residence times [7]. Silica particle formation was also demonstrated by Khan et al. in a microfluidic device [8]. However, this work produced larger silica particles in sub-micrometer in diameter. On the other hand, post-processing of nanoparticles has not been shown in literature yet. Here we present the step towards developing a process that can be used for coating of quantum dots in a microfluidic reactor. We obtained monodisperse silica nanoparticles at the sizes comparable to batch-wise synthesis techniques and optimized the process to generate tens of nanometer in diameter sizes.

The silicon reactor was designed for the controlled synthesis of nanomaterials and it was previously used for $\mathrm{TiO}_{2}$ nanoparticle synthesis [7]. Here we use this reactor to generate silica nanoparticles and optimize the process so that later we can use this for quantum dot coating. The silicon microreactor (Fig 1) was designed to have a droplet based flow; where droplets of reagents are generated and later carried in a long processing channel by a carrier fluid. Silica layers were formed as the reagents mixed inside the droplets.

To investigate the optimum parameters for silica coating of quantum dots, the formation process of silica nanoparticles has been examined for different residence times and flow rate ratios 
between the droplet phase and the carrier phase. These parameters will later be used as optimum quantum dot coating parameters.

\section{METHODS}

\section{Microreactor Design}

The image of our microreactor is shown in Figure 1. The reactor has a droplet generation unit and a channel in which the reactions take place due to the mixing of the reagents carried inside the droplets. It has two outlets (3 and 4) so that the residence time of the reaction can be controlled. The reactor is made in silicon substrate for obtaining chemical robustness. And conventional silicon fabrication techniques were used to fabricate it. The silicon reactor was prepared with a channel depth and widths of $200 \mu \mathrm{m}$. Further details of the reactor are described in [7].

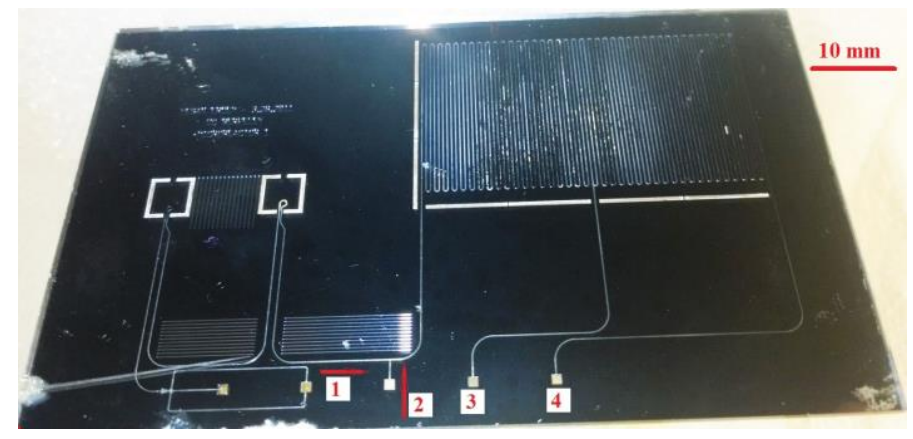

FIGURE 1: Image of Microreactor. $1 \& 2$ show the inlets for carrier fluid and reagents respectively, while $3 \& 4$ are the two outlets to examine the effect of different residence time [7].

\section{Materials}

For silica formation inside the microreactor, two reagents were prepared separately and N-methyl formamide (NMF) was used as the carrier fluid to induce the droplet generation at the Tjunction. To prepare the reagent 1 , we mixed $1.3 \mathrm{ml}$ of IGEPAL CO-520 with $10 \mathrm{ml}$ of anhydrous cyclohexane and stirred the solution for $15 \mathrm{~min}$. Finally, $80 \mu \mathrm{l}$ of tetraethylorthosilicate (TEOS) was added, followed by $15 \mathrm{~min}$ of stirring. Reagent 2 was prepared by adding $1500 \mu \mathrm{l}$ of ammonium hydroxide $(28 \%$ in water) to $10 \mathrm{ml}$ of anhydrous cyclohexane.

\section{Experimental Setup}

The experimental set-up consists of three syringe pumps to supply the carrier fluid and reagents through capillary tubing. The reagents were mixed inside the microchannels and as the carrier fluid was supplied through the main channel, droplets are formed at the T-junction. We have modified the batch-wise reaction described in $[9,10]$ to be used in the microreactor. As the ammonia in reagent 2 is mixed with reagent 1 containing
TEOS and surfactant, silica precursor starts to decompose and TEOS is hydrolyzed and replaces all IGEPAL. This ligand replacement leads to the formation of silica particles by reverse micelle in the microreactor.

\section{Procedure}

The formation of silica shells is induced by the presence of ammonia in the mixture of reagents; however, as the ammonia is soluble in the carrier fluid, therefore the precise investigation on the effect of ammonia concentration has a great level of importance to enhance the silica coating. All reactions were completed at the room temperature to maintain similar conditions to the batch-wise synthesis. The control parameters for the reaction in the microreactor were chosen as the residence time and the concentration of reagents. The residence time was precisely controlled by adjusting the flow rate of the carrier fluid, considering a constant length of the channel in the fabricated microreactor $(2 \mathrm{~m})$. The concentration of ammonia was controlled by both changing the flow rate ratios in the mixture of reagents and changing the concentration of the main solution in reagent 2 . Throughout the experiment, the flow rate ratios between reagents 1 and 2 were kept constant at 1:1 so the concentration in the main solution remained as the only controlling parameter.

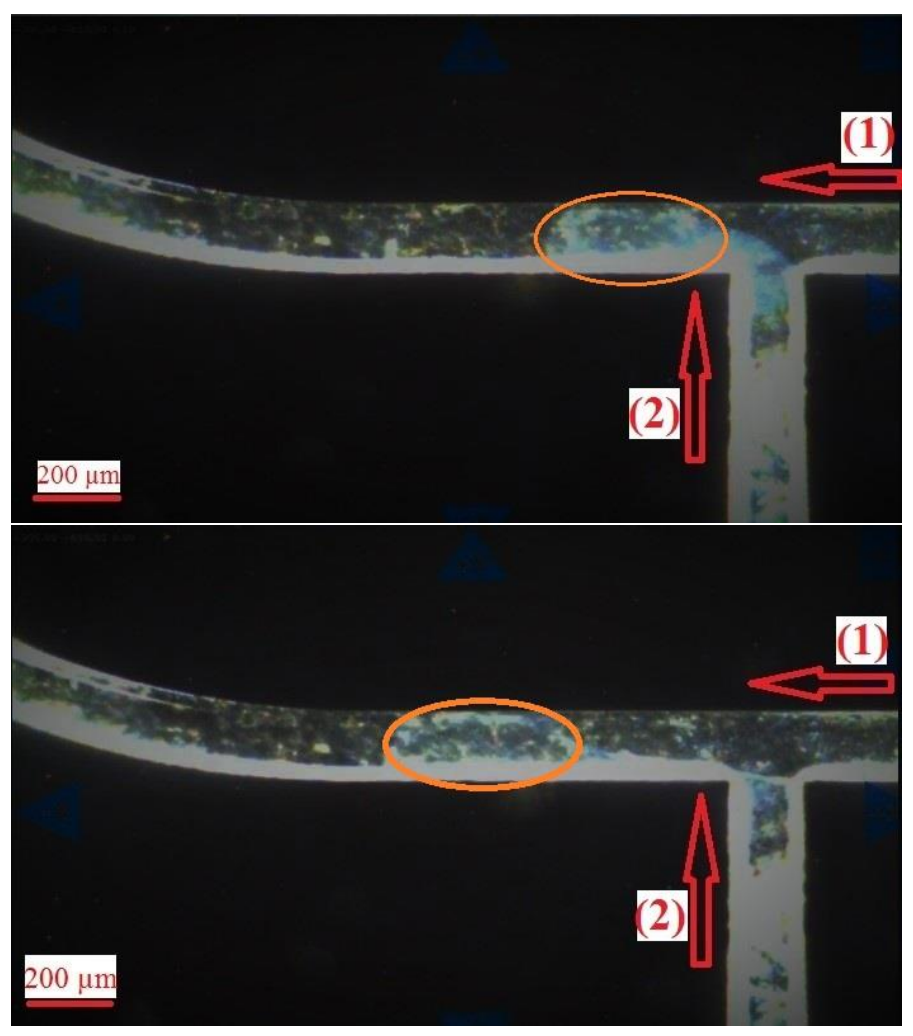

FIGURE 2: Droplet generation images at the T-junction. Arrow 1 shows the flow direction of NMF as the carrier fluid with a flow rate of $9.5 \mu \mathrm{l} / \mathrm{min}$. Arrow 2 indicates the direction of reagents with a total flow rate of $4.0 \mu \mathrm{l} / \mathrm{min}$. 
All the experiments were performed without the quantum dot cores, just to maintain and optimize the parameters for silica coating of quantum dots. In our future studies, the quantum dots will be supplied through reagent 1 and the same synthesis will be performed. To ensure uniformity of the reaction in droplets; the T-junction and the microchannels of the reactor were monitored online. At the optimum flow rates, the droplets were generated constantly with similar size range at the T-junction as shown in Figure 2. Based on various experiments and investigation on the controlling parameters, in order to reach to a size range close to the one obtained by the batch-wise synthesis, the flow rates were adjusted as $2.0 \mu \mathrm{l} / \mathrm{min}$ for the reagents and $9.5 \mu \mathrm{l} / \mathrm{min}$ for the carrier fluid. Residence time was kept around 260 seconds. The samples obtained from the reactor were immediately centrifuged to eliminate the effect of excess ammonia on particle formation.

\section{RESULTS AND DISCUSSION}

TEM images of the silica nanoparticles obtained by the optimum flow rates are shown in Figure 3. As illustrated in the TEM results, the average diameter of the silica shells has reached $25 \pm 2.7 \mathrm{~nm}$. Moreover, the monodispersity of the silica shells agrees with the initial idea about using the droplet-based microreactors for nanoparticle processing. If we compare this reaction with batch-wise synthesis methods that take about 48 hours, the microreactor reactions took a much shorter reaction time ( $460 \mathrm{~s})$ and much smaller amount of solutions were needed for the process. We have reached comparable results with the conventional batch-wise synthesis methods with higher efficiency.

The EDX results also show the existence of silica in the sample as shown in Figure 4. Since TEM grids were made out of $\mathrm{Cu}$, it also appears as a high peak in the graph. Based on the monodispersity and size range obtained, this method could be implemented for silica coating of quantum dots when these particles were added in the reagent solutions.

\section{CONCLUSION}

The droplet-based method introduced here, enables the growth of silica particles inside the reactor with comparable size range and monodispersity to batch-wise techniques. Moreover, shorter processing time and the decrease amount of materials used offer a huge advantage compared to the batchwise synthesis. The precise control over the residence time, concentration and temperature, could be used as a rapid and efficient method for silica coating of quantum dots. The next step in this study is to supply the $\mathrm{CdSe} / \mathrm{CdS}$ core/shell quantum dots in the reagents and study the formation of the silica shell around the cores.
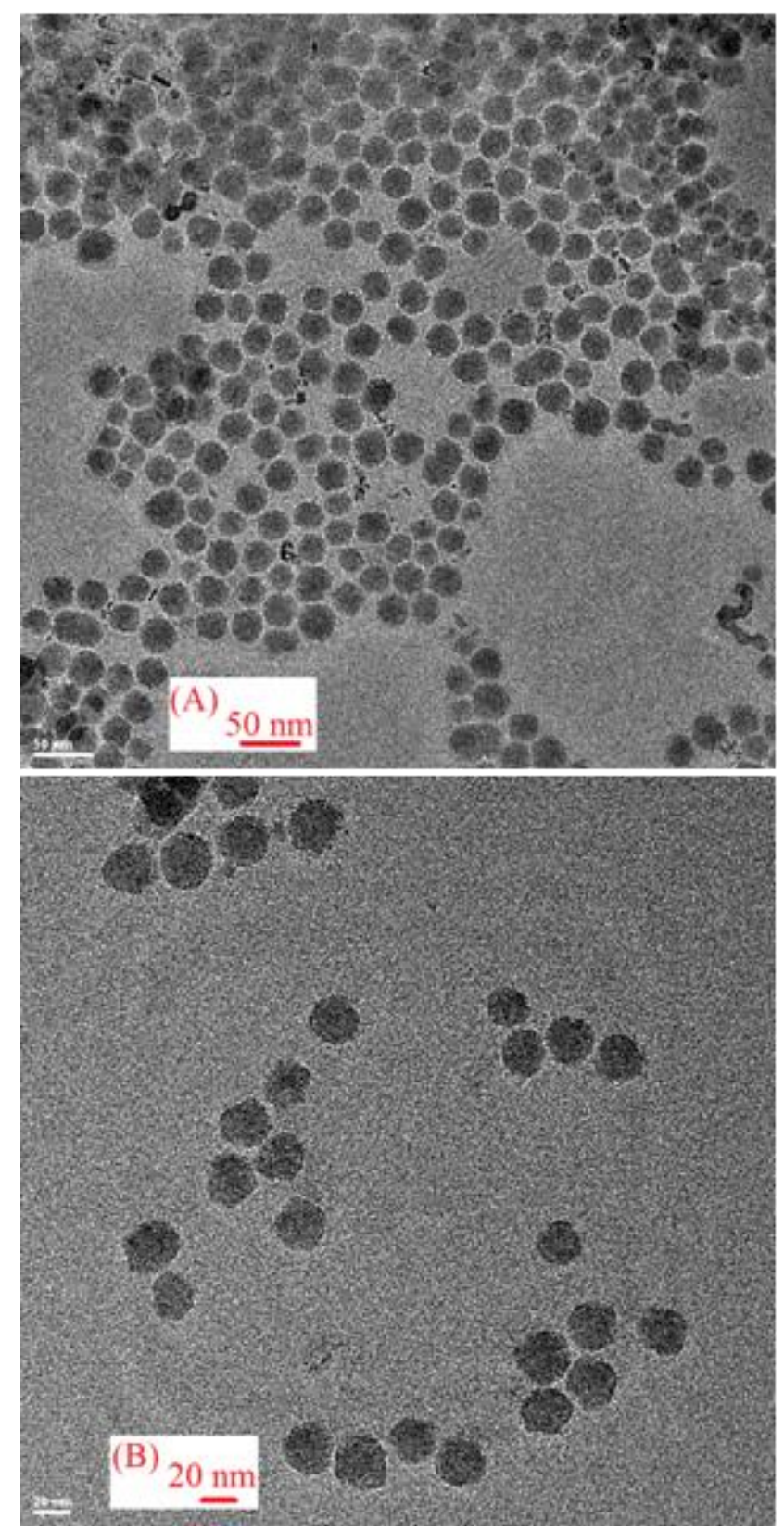

FIGURE 3: TEM results for silica shell growth inside the reactor. The monodisperse particles have a diameter size range of $25 \pm 2.7 \mathrm{~nm}$. Flow rates for carrier fluid and reagents are $9.5 \mu \mathrm{l} / \mathrm{min}$ and $2.0 \mu \mathrm{l} / \mathrm{min}$ respectively. 




FIGURE 4: EDX result for the particles shown in Figure 3. The low percentage of Silica (Silicon and Oxygen) is mainly due to the flow rate ratios assigned in the experiment.

\section{ACKNOWLEDGMENTS}

We would like to acknowledge Mr. Mustafa Guler for his help with the TEM usage of the National Nanotechnology Center (UNAM) clean room facilities.

\section{REFERENCES}

[1] I. Gorelikov, A. Martin, M. Seo, N. Matsuura, Silica-coated Quantum Dots for Optical Evaluation of Perfluorocarbon Droplet Interactions with Cells, Langmuir, vol. 27, pp.

1502415033, 2011.

[2] Y. Ma, Y. Li, S. Ma, X. Zhonget, Highly Bright WaterSoluble Silica Coated Quantum Dots with Excellent Stability, Journal of Materials Chemistry B, vol. 2, pp. 50435051,

2014.

[3] D. K. Yi, S. T. Selvan, S. S. Lee, G. C. Papaefthymiou, D. Kundaliya, J. Y. Ying, Silica-Coated Nanocomposites of Magnetic Nanoparticles and Quantum Dots, Journal of American Chemisty Society, vol. 127, pp. 4990-4991, 2005.

[4] L. Qian et al, Photoluminescene from silica coated CdSe quantum dots, Applied Physics Letter vol. 94(7), 2009.

[5] Bryan D. Andersom, W.C. Wu, and J.B. Tracy, Silica overcoating of CdSe/CdS Core/Shell Quantum Dot Nanorods with Controlled Morphologies, Chem. Mater, 28, pp. 49454952, 2016.

[6] W. Stober, A. Fink, and E. Bohn, Controlled Growth of Monodisperse Silica Spheres in the Micron Size Range, Journal of Colloid and Interface Science, 26, pp. 62-69, 1968.
[7] E. Y. Erdem et al. Droplet-based Microreactor for Increased Temperature Control in Nanoparticle Synthesis, Small,10, No. 6, pp. 1076-1080, 2014.

[8] S. A. Khan, A. Günther, M. A. Schmidt, and K. F. Jensen, "Microfluidic synthesis of colloidal silica," Langmuir, 20: 8604-8611, 2004.

[9] Z. Popović et al., A Nanoparticle Size Series for In Vivo Fluorescence Imaging, Angewandte Chemie, vol. 49, no. 46, pp. 8649-52, Nov. 2010.

[10] A. Guerrero-Martinzez et al., Recent Progress on Silica Coating of Nanoparticles and Related Nanomaterials, Advanced Mater, 22, pp. 1182-1195, 2010. 